\title{
Validación de una medida estandarizada en la longitud de inserción del catéter venoso central yugular para la posicion óptima de su punta
}

\section{Validation of a standardized measurement in the insertion length of the central jugular venous catheter for the optimal positioning of its tip}

\author{
Riky L. Pérez-Lucas*, Jorge Torres-Martínez, Viridiana R. Lara-González, \\ Jesús H. Rivera-Bañuelos, Miguel A. Lara-Pérez, José A. Reyes-Monroy y Julio A. Serrano-Lozano
}

Servicio de Angiología y Cirugía Vascular, Hospital Regional Lic. Adolfo López Mateos, Instituto de Seguridad y Servicios Sociales de los Trabajadores del Estado (ISSSTE), Ciudad de México, México

\begin{abstract}
Resumen
Objetivo: Obtener una medida de longitud de inserción del catéter venoso central para la colocación óptima de su punta. Material y métodos: Estudio prospectivo no aleatorizado de julio de 2019 a marzo de 2020. Se agruparon en dos grupos con distinto abordaje de sitio de inserción. Se compararon los paciente cuyos catéteres fueron colocados mediante la técnica con medida estandarizada y el grupo control, cuya longitud de inserción fue a criterio del médico que realizó el procedimiento, posteriormente se verificó la posición de la punta en radiografía de tórax. Se realizó el análisis estadístico con significancia estadística de $<0.05$. Resultados: Hubo un total de 134 pacientes que requirieron colocación de un catéter venoso central, 55 se sometieron a la técnica con medida estandarizada y 79 fueron controles. En el grupo con sitio de inserción yugular derecho se comparó al subgrupo sometido a la técnica con medida estandarizada contra el grupo control, y se encontró un RR de 5.9 (IC 95\%: 3.0-11.5; $p$ < 0.001) para presentar posición óptima. En el sitio de inserción yugular izquierdo se encontró un RR de 1.9 (IC 95\%: 1.0-3.5; $p=0.02$ ). Conclusiones: El uso de la técnica con medida estandarizada pronostica una posición óptima de la punta de catéter venoso central en sitio yugular.
\end{abstract}

Palabras clave: Cateterización. Vena central. Procedimiento endovascular. Catéter venoso central.

\begin{abstract}
Objective: To obtain a measurement of the insertion length of the central venous catheter for optimal placement of its tip. Material and methods: Prospective non-randomized study from July 2019 to March 2020; patients were grouped into two groups with different insertion site approach, the patients whose catheters were placed using the standardized measurement technique and the control group whose insertion length was a criterion of the physician who performed the procedure were compared, then the position was verified of the tip on chest radiograph. Statistical analysis was performed with statistical significance of <0.05. Results: Of a total of 134 patients who required the placement of a central venous catheter, 55 patients underwent the technique with standardized measurement and 79 patients were controls. In the group with the right jugular
\end{abstract}


insertion site, the subgroup subjected to the measurement was compared against the control group and a $R R$ of 5.9 (95\% $\mathrm{Cl} 3.0-11.5, p<0.001)$ was found to present optimal position. In the group with the left jugular insertion site, a RR of 1.9 was found (95\% Cl 1.0-3.5, $p=0.02)$. Conclusions: The use of a standardized measure predicts an optimal position of the central venous catheter tip in the jugular site.

Key words: Catheterization. Central vein. Endovascular procedure. Central venous catheter.

\section{Introducción}

En México, del 85 al 90\% de los pacientes que ingresan a un centro hospitalario requieren de un acceso vascular, ya sea periférico o central, que expone al paciente a presentar algún tipo de evento adverso relacionado con el manejo del sistema integral de terapia intravenosa ${ }^{1}$.

El catéter venoso central (CVC) desempeña un papel muy importante en el tratamiento de pacientes críticamente enfermos, tanto en la unidad de terapia intensiva como en hospitalización, ya que se requiere para la infusión de fluidos no hipertónicos y el monitoreo de la presión venosa central ${ }^{2}$. La posición no óptima de la punta del catéter es una razón común para la falla prematura de los CVC debido a la trombosis de la vena y/o el catéter, a su vez, esto condiciona el desarrollo de estenosis venosa central ${ }^{3,4}$.

Los CVC insertados a una profundidad inadecuada podrían quedar fácilmente fuera de la vena cava superior (VCS), lo que podría aumentar el riesgo de formación de trombos y/o infección ${ }^{5}$. La inserción excesiva del catéter también puede provocar diversas arritmias, como latidos prematuros auriculares y ventriculares, taquicardia o fibrilación ventricular. Estas alteraciones del ritmo son generalmente resistentes a la supresión por fármacos y requieren de la extracción del catéter de las cámaras cardíacas ${ }^{6}$.

Las complicaciones mecánicas incluyen taponamiento cardíaco, perforación cardíaca, perforación de la pared vascular, así como erosiones cardíacas, daño a la válvula tricúspide, arritmias ventriculares y auriculares, neumotórax y hemotórax?

En un estudio, Peres utilizó la altura de los pacientes para desarrollar fórmulas para predecir la longitud adecuada del catéter que se insertará en la vena yugular derecha (VYD), en la vena yugular izquierda (VYI), y en la vena subclavia (VSC) derecha e izquierda, sin embargo, no logra obtener una posición óptima de la punta del catéter ${ }^{8}$.

Idealmente, la punta del catéter se debe localizar en la unión de la vena cava superior y la reflexión pericárdica. Esta ubicación garantiza una posición proximal con alto flujo sanguíneo que previene la trombosis, además, esta se encuentra fuera de la aurícula y, por lo tanto, evita arritmias causadas por la irritación de la punta del catéter de la pared auricular derecha ${ }^{9}$.

El límite superior de la reflexión pericárdica no se puede ver en la radiografía simple de tórax, pero generalmente se acepta que esté aproximadamente a $0,8 \mathrm{~cm}$ por debajo de la carina y el límite inferior a $3 \mathrm{~cm}$ por debajo de la carina. Esto ha sido evaluado en cadáveres conservados y frescos. Además, es fácilmente visible incluso en una radiografía de tórax anteroposterior portátil de baja calidad ${ }^{10}$.

Por lo tanto, la carina ha sido considerada como un hito radiológico para la posición de la punta del CVC (Fig. 1). Las zonas A y B mencionadas en la figura están en relación con la carina. Estas zonas son guías útiles, sin embargo, los catéteres de uso prolongado deben colocarse con precisión bajo fluoroscopia, la posición de la punta del catéter de uso corto debe confirmarse con una radiografía de tórax posterior al procedimiento y para considerarse en posición óptima debe encontrarse a $2 \mathrm{~cm}$ de la carina ${ }^{11,12}$.

La distancia promedio desde la VYD a la reflexión pericárdica es de $16 \mathrm{~cm}$, sin embargo, se encontró que en 7 de $38(16 \%)$ catéteres con longitud de inserción de $16 \mathrm{~cm}$ colocados a través de la VYD, la punta eran intracardiaca ${ }^{13}$.

Los catéteres del lado izquierdo son más complejos, puesto que presentan dos angulaciones, además de una longitud corta de VCS. La distancia al corazón desde la VYI es de entre $19 \mathrm{~cm}$ en promedio ${ }^{14}$. Sin embargo, es común la angulación aguda de la punta del CVC, ya que se apoya en la pared lateral derecha de la VCS. Una revisión reportó que el sitio de inserción yugular izquierdo con longitud de inserción de 16 $\mathrm{cm}$ de largo, ninguno era intracavitario, usando la carina como marcador ${ }^{15}$.

Se realizaron estimaciones de acuerdo a un trabajo publicado indicando que, si los CVC se colocan a 13 $\mathrm{cm}$, se podría estar seguro que, en un $95 \%$ de los CVC, la punta del catéter estaría extracardiaca ${ }^{16}$.

No encontramos estudios prospectivos que usen alguna técnica con medida estandarizada por género y sitio de inserción, por lo que la técnica con medida 


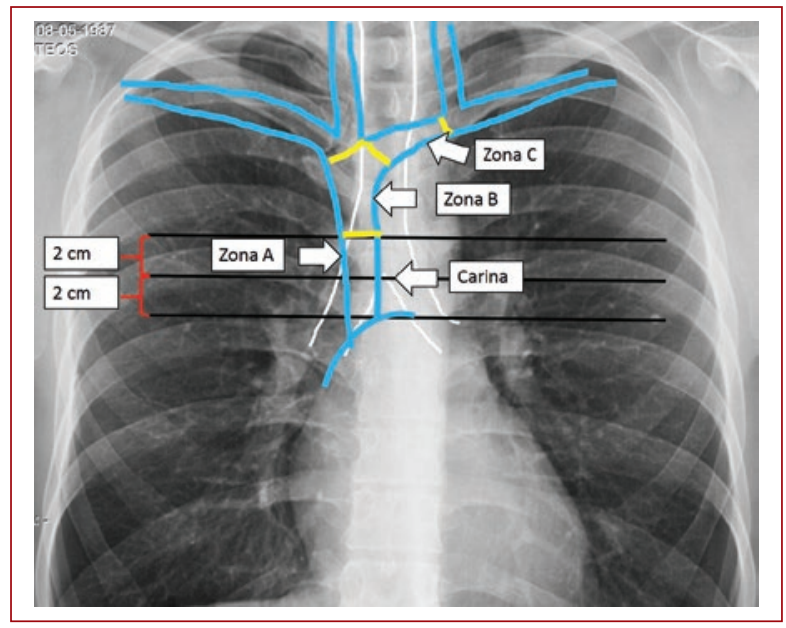

Figura 1. Zonas $A, B$ y $C$ donde se puede presentar la punta del catéter venoso central. Zona $A$, posición optima de punta. En blanco, la tráquea y los bronquios; en azul, la vena cava superior.

estandarizada propuesta no ha sido estudiada. Por ello se propone el uso de una técnica con medida estandarizada (una longitud de inserción determinada) para su uso práctico y para evitar el uso equipos costosos, estudios previos o fórmulas, y así disminuir la tasa de recolocación y sus complicaciones de la punta de catéter.

\section{Material y métodos}

Se realizó un estudio prospectivo no aleatorizado en el Hospital Regional Lic. Adolfo López Mateos donde se incluyó a todos los pacientes sometidos a la colocación de CVC guiado con ultrasonido en sitio de inserción yugular derecho e izquierdo por el servicio de Angiología y Cirugía Vascular de julio 2019 a marzo 2020. A los pacientes se les explicó riesgo y beneficio de la colocación de catéter venoso central y firmaron el consentimiento informado con respecto a la colocación de catéter venoso central. Además, los que aceptaron participar firmaron consentimiento informado para el estudio.

Se colocaron los CVC en el sitio de la vena yugular interna, tanto en el lado derecho como en el lado izquierdo, por la técnica de inserción central; bajo visión con ultrasonido se localizó la bifurcación del músculo esternocleidomastoideo (ECM) y se insertó la aguja justo caudal a su vértice en un ángulo de $30^{\circ}$, apuntando hacia el pezón ipsilateral.

Se incluyó a pacientes mayores de 18 años que requirieron un acceso central de primera instancia en la vena yugular. Se excluyó a pacientes que requirieron abordaje en la vena subclavia o femoral, pacientes que fueron sometidos a otra técnica de inserción en la yugular que no fuera la de abordaje central, pacientes que reusaron a la colocación del CVC, pacientes que requirieron colocación en la sala de fluoroscopio. Se eliminaron pacientes que no contaban con radiografía valorable posterior a la intervención y pacientes cuya punta de CVC fuera extratorácica.

Se dividió el total de pacientes respecto al sitio de inserción yugular en el lado derecho y en el lado izquierdo, y se propuso una medida estandarizada que correspondía a la longitud de inserción en cada sitio de abordaje, tomando en cuenta el género del paciente. La maniobra principal fue comparar la posición óptima de la punta del catéter venoso central (en la vena cava superior), adoptando una técnica con medida estandarizada, que corresponde en el sitio de inserción yugular derecho a $13 \mathrm{~cm}$ en hombres y $12 \mathrm{~cm}$ en mujeres, y en el sitio de inserción yugular izquierdo a $14 \mathrm{~cm}$ en hombres y $13 \mathrm{~cm}$ en mujeres. La posición óptima de la punta de estos catéteres se comparó con los que no fueron sometidos a la técnica con medida estandarizada, es decir, a criterio del médico que lo colocó.

En ambos grupos se definió como colocación óptima de la punta del catéter como la distancia dentro de $2 \mathrm{~cm}$ cercana a la carina, ya sea superior o inferior, verificada con la radiografía posterior a la colocación del CVC.

En cada subgrupo con sitio de inserción (derecho e izquierdo) se comparó, dividido por género, la colocación óptima de la punta con la técnica de medida estandarizada contra la colocación sin la técnica con medida estandarizada. El análisis estadístico se realizó con la prueba Fisher y se determinó su significancia estadística en $<0.05$, además se calculó riesgo relativo de probabilidad de colocación de punta de catéter en cada grupo con sitio de inserción.

\section{Resultados}

Se encontró una edad media de 64.2 años en el grupo sin la técnica con medida estandarizada y 63.7 años en el grupo con la técnica con medida estandarizada (Tabla 1). Se encontró menor proporción de colocación de punta óptima en el grupo sin la técnica con medida estandarizada (18.5\%) comparada con el grupo con la técnica con medida estandarizada (75.8\%). El promedio de longitud de inserción en el grupo control fue de $15.2 \mathrm{~cm}$ contra $12.7 \mathrm{~cm}$ en el grupo en el que se utilizó la técnica con medida 
Tabla 1. Datos demográficos de los dos grupos

\begin{tabular}{|l|c|c|}
\hline & $\begin{array}{c}\text { Técnica sin medida estandarizada } \\
\text { (otras técnicas) }\end{array}$ & $\begin{array}{c}\text { Técnica con medida } \\
\text { estandarizada }\end{array}$ \\
\hline Número de pacientes (N) & 81 & 62 \\
\hline Edad (media, DE) & $64.2( \pm 11.3)$ & $63.7( \pm 15.1)$ \\
\hline Mujeres (\%) & $39(48.1 \%)$ & $28(45.1 \%)$ \\
\hline Yugular derecha (n, \%) & $65(79.2 \%)$ & $46(74.1 \%)$ \\
\hline Yugular izquierda (n, \%) & $16(19.7 \%)$ & $14(22.5 \%)$ \\
\hline Posición óptima & $15(18.5 \%)$ & $47(75.8 \%)$ \\
\hline Longitud de inserción (media cm, DE) & $15.2( \pm 0.9)$ & $12.7( \pm 0.6)$ \\
\hline Distancia de la carina a la punta (media cm, DE) & $4.0( \pm 2.0)$ & $1.4( \pm 1.6)$ \\
\hline
\end{tabular}

DE: desviación estándar

estandarizada. La distancia de la carina a la punta fue mayor en el grupo control $(4 \mathrm{~cm})$, comparado con el grupo con técnica con medida estandarizada $(1.4 \mathrm{~cm})$.

En el estudio en que se abordó a los pacientes sometidos al sitio de inserción yugular derecho, se comparó la técnica con medida estandarizada contra otras técnicas a criterio del médico. La técnica con medida estandarizada presentó un aumento de 5.9 RR para la colocación óptima de la punta (Tabla 2). En el subgrupo de mujeres con sitio de inserción yugular derecho a las que se les aplicó la técnica con medida estandarizada se asoció con un aumento de 6.5 RR de probabilidad de colocación óptima de la punta de catéter. En el subgrupo de hombres con sitio de inserción yugular derecho a los que se les aplicó la técnica con medida estandarizada presentó 5 RR más frecuente la colocación óptima de la punta de catéter.

En los pacientes a los que se abordó el sitio de inserción yugular izquierdo, al compararse la técnica con medida estandarizada, se encontró 1.9 RR veces más frecuente la posición óptima usando la técnica medida estandarizada (Tabla 3). En él subgrupo de mujeres con sitio de inserción yugular izquierdo se encontró 3 RR veces una posición óptima de la punta de catéter a las que se les aplicó la técnica con medida estandarizada. En el subgrupo masculino fue 1.3 RR veces más frecuente en el grupo expuesto a la técnica con medida estandarizada, sin significancia estadística.

\section{Discusión}

La posición de la punta de CVC está asociada con algunas complicaciones, con una incidencia que varía del 6.2 al $11.8 \%$ en sitios de inserción de la vena
Tabla 2. Comparación de la técnica con medida estandarizada en el sitio de inserción en la vena yugular derecha

\begin{tabular}{|l|c|c|c|c|}
\hline CVC en VYD & Óptima & $\begin{array}{c}\text { No } \\
\text { óptima }\end{array}$ & $\begin{array}{c}\text { RR } \\
\text { (IC del 95\%) }\end{array}$ & ${ }^{*} \mathbf{p}$ \\
\hline Estandarizada & $\mathbf{n}$ & $\mathbf{n}$ & & \\
\hline No estandarizada & 35 & 13 & $5.9(3.0-11.5)$ & $<0.001$ \\
\hline $\begin{array}{l}\text { CVC en VYD en mujeres } \\
\text { Estandarizada }\end{array}$ & 15 & 57 & & \\
\hline $\begin{array}{c}\text { No estandarizada } \\
\text { CVC en VYD en hombres }\end{array}$ & 4 & 26 & $6.5(2.4-17.3)$ & $<0.001$ \\
\hline $\begin{array}{c}\text { Estandarizada } \\
\text { No estandarizada }\end{array}$ & 20 & 6 & $5(1.9-12.5)$ & $<0.001$ \\
\hline
\end{tabular}

CVC: catéter venoso central; IC: intervalo de confianza; p: valor de significancia; RR: riesgo relativo; VYD: vena yugular derecha.

Tabla 3. Comparación de la técnica con medida estandarizada en el sitio de inserción en la vena yugular izquierda

\begin{tabular}{|c|c|c|c|c|}
\hline \multirow[t]{2}{*}{ CVC en VYI } & Óptima & $\begin{array}{c}\text { No } \\
\text { óptima }\end{array}$ & $\begin{array}{c}\text { RR } \\
\text { (IC del 95\%) }\end{array}$ & \multirow[t]{2}{*}{${ }^{*} p$} \\
\hline & $\mathbf{n}$ & $\mathbf{n}$ & & \\
\hline Estandarizada & 12 & 2 & $1.9(1.0-3.5)$ & 0.021 \\
\hline No estandarizada & 7 & 9 & & \\
\hline $\begin{array}{l}\text { CVC en VYI en mujeres } \\
\text { Estandarizada } \\
\text { No estandarizada }\end{array}$ & $\begin{array}{l}5 \\
3\end{array}$ & $\begin{array}{l}0 \\
6\end{array}$ & $3(1.1-7.5)$ & 0.028 \\
\hline $\begin{array}{l}\text { CVC en VYI en hombres } \\
\text { Estandarizada } \\
\text { No estandarizada }\end{array}$ & $\begin{array}{l}7 \\
4\end{array}$ & $\begin{array}{l}2 \\
3\end{array}$ & $1.3(0.6-2.8)$ & $\begin{array}{c}0.70 \\
\text { (NS) }\end{array}$ \\
\hline
\end{tabular}

CVC: catéter venoso central; IC: intervalo de confianza; NS: No significativo; p: valor de significancia; RR: riesgo relativo; VYI: vena yugular izquierda. 
subclavia y la vena yugular interna. Esas complicaciones incluyen perforación cardíaca, tamponade cardíaco, perforación de la pared venosa, erosión cardíaca, daño de la válvula tricúspide, neumotórax, hemotórax, arritmias ventriculares y atriales ${ }^{2}$.

La posición subóptima o no óptima de la punta del CVC es relativamente común durante la colocación de catéteres sin examen de rayos $X$. En la mayoría de las circunstancias, si el CVC está mal posicionado o torcido, debe ser recolocado, reemplazado o retirado tan pronto como sea posible ${ }^{3}$.

En un estudio retrospectivo no publicado y realizado en el Hospital Regional Lic. Adolfo López Mateos se encontró que del total $(n=690)$ de CVC colocados de enero a junio del 2019 , el $31 \%$ presentaron la punta del catéter en posición óptima.

Por otra parte existe el riesgo de que la punta del CVC esté por fuera de la VCS que incrementaría el potencial de formación de trombo. Varias recomendaciones se han propuesto para disminuir el riesgo de perforación de vaso y cardíaca que incluyen evitar el lado izquierdo e inmovilización del catéter. Sin embargo, el método más efectivo que se ha encontrado es la colocación de la punta en el sitio extracardiaco en VCS y su confirmación con radiografía de tórax ${ }^{15}$.

Se ha demostrado que una profundidad de $16 \mathrm{~cm}$ para el sitio de inserción en la yugular izquierda (YI) puede ser adecuada para la población estadounidense, pero la población mexicana puede requerir menor longitud de inserción².

La ausencia de una correlación significativa entre la estatura y la longitud de inserción sugiere que la estatura por sí sola no es un factor para la óptima colocación de la punta del catéter, confirmado por estudios previos, por lo que una medida estandarizada sería mejor opción ${ }^{11-13}$.

El limitante de otras técnicas para mejorar la posición óptima del catéter es que requieren equipos especiales o incluso una radiografía de tórax previa para la medición, teniendo una utilidad limitada en la práctica clínica, el uso de la técnica con medida estandarizada se realizó sin requerir más estudios o incluso sin la estatura de los pacientes ${ }^{17}$.

Los hallazgos muestran que una colocación CVC en el sitio de inserción yugular derecho con la técnica con medida estandarizada de longitud de inserción puede ser usada en nuestra población adulta, ya que disminuye la probabilidad de colocación de la punta de catéter en posición óptima confirmada por radiografía de tórax posterior a la intervención.
En este estudio, en el grupo con sitio de inserción yugular derecho, se encontró un aumento en la probabilidad de presentar una posición óptima de la punta del catéter usando una técnica con medida estandarizada de inserción, lo que previamente se había propuesto con esa medida. ${ }^{2}$

En el grupo con sitio de inserción yugular izquierdo no fue concluyente, ya que no alcanzó significancia estadística en la población masculina, probablemente por el tamaño de la muestra, por lo que se requeriría un mayor tamaño de la muestra. Por lo tanto, los datos obtenidos no se cumplen con la hipótesis relacionada con el grupo con sitio de inserción yugular izquierdo en hombres.

En relación con el objetivo principal planteado podemos afirmar que se compararon las poblaciones en estudio en los sitio yugular derecho e izquierdo y cumplió con los hallazgos ya mencionados, además se cumplió con el objetivo secundario de disminuir la incidencia de recolocación de catéter posterior a la verificación de su punta por radiografía de tórax en el grupo con sitio de inserción yugular derecho. Sin embargo, el estudio no evaluó las complicaciones relacionadas con la posición de la punta, por lo que se requeriría un nuevo estudio donde se incluyeran.

Este estudio carece de suficiente peso para realizar alguna recomendación a otras poblaciones, ya que no fue aleatorizado ni fue un estudio multicentrico. Además, el estudio no se puede aplicar a población pediátrica. Sería conveniente, además, comparar la medida estandarizada con fórmulas en sitios de inserción yugular y probablemente otro tipos de catéter, ya sea catéter para hemodiálisis y/o catéter tunelizados.

\section{Conclusiones}

Se encontró una mayor frecuencia de posición óptima de la punta del catéter venoso central por vía de inserción yugular derecha, valorado por radiografía de tórax posterior a la intervención, con el uso de la técnica con medida estandarizada comparada con el uso de otras técnicas a criterio del médico. En el sitio de inserción de la yugular izquierda no se encontró significancia estadística cuando se compara la técnica con medida estandarizada contra el uso de otras técnica a criterio de médico propuesta en hombres, probablemente por el tamaño de la muestra.

Se sugiere el uso de la técnica con medida estandarizada en colocación de CVC en sitio de inserción yugular derecho ya que mejorará la posición óptima de la punta sin requerir formula o estudios especiales y disminuye número de recolocación. 


\section{Agradecimiento}

Los autores agradecen al personal de la clínica de catéteres del Hospital Regional Lic. Adolfo López Mateos el cumplimiento de normas éticas de acuerdo con las directrices de la institución.

\section{Conflicto de intereses}

Los autores declaran que no tienen conflicto de intereses.

\section{Financiamiento}

El equipo y material es propio del hospital, a los cuales tienen derecho los pacientes que participaron en el estudio. No contó con financiamiento ajeno a la institución.

\section{Responsabilidades éticas}

Protección de personas y animales. Los autores declaran que para esta investigación no se han realizado experimentos en seres humanos ni en animales.

Confidencialidad de los datos. Los autores declaran que han seguido los protocolos de su centro de trabajo sobre la publicación de datos de pacientes.

Derecho a la privacidad y consentimiento informado. Los autores declaran que en este artículo no aparecen datos de pacientes.

\section{Bibliografía}

1. Escamilla JJ, Ramírez GI. Uso de kits desechables para optimizar tiempos, movimientos y apego a protocolos de la terapia intravenosa. Rev Cuid. 2017;8(3):1749-57.

2. Kujur R, Rao MS, Mrinal M. How correct is the correct length for central venous catheter insertion. Indian J Crit Care Med. 2009;13(3):159-62.

3. Gibson F, Bodenham A. Misplaced central venous catheters: applied anatomy and practical management. Br J Anaesth. 2013;110(3):333-46.

4. McGee DC, Gould MK. Preventing complications of central venous catheterization. N Engl J Med. 2003;348(12):1123-33.

5. Sivasubramaniam S, Hiremath M. Central Venous Catheters: Do we need to Review Practice on Positioning? J Intensive Care Soc. 2008:9(3):228-31.

6. Kasten GW, Owens E, Kennedy D. Ventricular tachycardia resulting from central venous catheter tip migration due to arm position changes: report of two cases. Anesthesiology. 1985;62(2):185-7.

7. Fletcher SJ, Bodenham AR. Safe placement of central venous catheters: where should the tip of the catheter lie? Br J Anaesth. 2000;85(2):188-91.

8. Peres PW. Positioning central venous catheters--a prospective survey. Anaesth Intensive Care. 1990;18(4):536-9.

9. Pikwer A, Bååth L, Davidson B, Perstoft I, Akeson J. The incidence and risk of central venous catheter malpositioning: a prospective cohort study in 1619 patients. Anaesth Intensive Care. 2008;36(1):30-7.

10. Andrews RT, Bova DA, Venbrux AC. How much guidewire is too much? Direct measurement of the distance from subclavian and internal jugular vein access sites to the superior vena cava-atrial junction during central venous catheter placement. Crit Care Med. 2000;28(1):138-42.

11. Albrecht K, Nave H, Breitmeier D, Panning B, Tröger HD. Applied anatomy of the superior vena cava-the carina as a landmark to guide central venous catheter placement. Br J Anaesth. 2004;92(1):75-7.

12. Stonelake PA, Bodenham AR. The carina as a radiological landmark for central venous catheter tip position. Br J Anaesth. 2006;96(3):335-40.

13. McGee WT, Ackerman BL, Rouben LR, Prasad VM, Bandi V, Mallory DL. Accurate placement of central venous catheters: a prospective, randomized, multicenter trial. Crit Care Med. 1993;21(8):1118-23.

14. Andrews RT, Bova DA, Venbrux AC. How much guidewire is too much? Direct measurement of the distance from subclavian and internal jugular vein access sites to the superior vena cava-atrial junction during central venous catheter placement. Crit Care Med. 2000;28(1):138-42.

15. Schuster M, Nave H, Piepenbrock S, Pabst R, Panning B. The carina as a landmark in central venous catheter placement. $\mathrm{Br} \mathrm{J}$ Anaesth. 2000; 85(2):192-4

16. Russell WC, Parker JL. Thirteen centimetre central venous catheters, lucky for all? Anaesthesia. 2003;58(4):388

17. Czepizak CA, O'Callaghan JM, Venus B. Evaluation of Formulas for Optimal Positioning of Central Venous Catheters. 1995;107(6):1662-4. 